\title{
On-farm Production of Arbuscular Mycorrhizal (AM) Fungi Using Trap Crop Cycles
}

\author{
S.B. Raut ${ }^{1 *}$, C.D. Deokar ${ }^{2}$, A.M. Navale ${ }^{1}$ and J.A. Dahatonde ${ }^{3}$ \\ ${ }^{1}$ Department of Plant Pathology and Agriculture Microbiology, \\ Post Graduate Institute, MPKV, Rahuri, India \\ ${ }^{2}$ Plant Pathology and Agriculture Microbiology Section, College of Agriculture, Dhule, India \\ ${ }^{3}$ Department of Agriculture Entomology, DVVPF's College of Agriculture, \\ Ahmednagar, India \\ *Corresponding author
}

\section{A B S T R A C T}

\section{Keywords \\ AM fungi, On-farm production, Nucleus inoculums and mass inoculum \\ Article Info \\ Accepted: \\ 10 September 2019 \\ Available Online: \\ 10 October 2019}

The method of preparation of mass inoculum (MI) of native AM fungi was standardized following the principle of multiplying the nucleus inoculum (NI) of AMF (consortium of native soils) under partially sterilized in situ. Inoculation of arbuscular mycorrhizal fungi in root zone soils of different agricultural crops for which treatment no. 17 (Fenugreek-MaizeSorghum) host trap crops was found to be suitable for their mass multiplication.

\section{Introduction}

The microbial communities have become an integral part of the biosphere. They play a key role in maintaining the biological equilibrium in biosphere. The living components developed certain relationships such as symbiotic, mutualistic and antagonistic. These relationships helped to maintain biological equilibrium of nature. The word Mycorrhiza (Greek: mykes $=$ mushroom; rhiza $=$ root $)$ was coined by Albert Bernard Frank in 1885. The
German Forest Plant Pathologist, to describe the mutual association of two different organisms, plant and fungus, which benefit from each other in a mutualistic symbiosis under ideal conditions, i.e. the plant provides carbohydrates for the fungus, which in turn makes nutrients available for the plant (Harley, 1959).

The inocula of AM fungi are commercially available, production of AM fungus inoculum on the farm is an attractive alternative. 
Purchasing the large amounts of inoculum necessary for large-scale agriculture may cost prohibitive. Producing the inoculum on-farm saves processing and shipping costs included in the price of commercial inocula. These factors are the primary reason why most onfarm methods have been utilized in developing nations. Another benefit of on-farm production of inoculum is that locally adapted isolates, which may be more effective than introduced ones in certain situations (Sreenivasa, 1992), can be produced when the farmers indigenous AMF communities are used as starter inocula.

\section{Materials and Methods}

\section{Laboratory instruments}

Different laboratory instruments used during the course of investigation were $\mathrm{pH}$ meter, electronic weighing balance, water bath, sieves $(500 \mu \mathrm{m}-250 \mu \mathrm{m}-125 \mu \mathrm{m}-105 \mu \mathrm{m}$ $75 \mu \mathrm{m}-45 \mu \mathrm{m})$ spectrophotometer, stereoscopic zooming microscope, compound microscope, microscope attached with digital camera, etc.

\section{Glassware}

Different types of Borosil make glassware, viz., petri dishes, glass slides, cover slips, pipettes, conical flasks and beakers of various capacities, glass rods, volumetric flasks, funnels, measuring cylinders of different capacities etc. were used.

\section{Chemicals}

The laboratory grade standard and pure chemicals used for study were $\mathrm{HgCl}_{2}, \mathrm{KOH}$, potassium dichromate, concentrated hydrochloric acid (HCL), phosphoric acid $\left(\mathrm{H}_{3} \mathrm{PO}_{4}\right)$, sodium fluoride $(\mathrm{NaF})$, ferrous sulphate, boric acid, potassium permanganate, sodium hydroxide, sulphuric acid $\left(\mathrm{H}_{2} \mathrm{SO}_{4}\right)$,
Darco-G 60, sodium bicarbonate, ammonium molybdate, stannous chloride solution etc.

\section{Miscellaneous materials}

These included spade, shovel, plastic ghamela, non-absorbent cotton, plastic pots, sand, F.Y.M., zip loc polythene bags, white labels, rubber bands, Whatman no.1 filterpaper, Whatman no.42 filter paper, buffer solutions of $\mathrm{pH}$ 7.0, 4.0 and 9.2, denatured spirit, ethyl alcohol, stains (cotton blue, lactophenol and acid fuchsin), polyvinyl alcohol-lactoglycerol (PVLG), Meltzer's reagent, ferroin indicator, hand instruments (cutter, inoculating needle, forceps), pencil, foot scale, test tube stand, glass marker, trap crop seeds viz., maize (Zea mays), fenugreek (Trigonella foenumgraecum), sorghum (Sorghum bicolor), pearl millet (Pennisetum typhoides L.) and coriander (Corriandrum sativum), LDPE mulching transparent sheet (25 micron), Arc GIS 10.2 software (for map preparation) etc.

\section{On-farm production technology}

\section{Preparation of Nucleus inoculum (NI)}

Nucleus inoculum of native AMF (consortium) was prepared by the following method. Surface sterilized (used 'Chloramin $\mathrm{T}$ ' for 1 minute) chlamydospores of native AMF consortium, predominated by Glomus spp., Aculospora spp., Sceutellospora spp., Rhizophagus spp. and Gigaspora albida isolated from natural root zone soils of eleven fruit crops from the central campus MPKV, Rahuri collected by wet sieving and decanting method were multiplied on sorghum (host plant) roots grown in 40 plastic pots filled with sterilized soil : sand : FYM mixture (1:1:1; v:v:v;) (SSF substrate) by inoculating with native AMF chlamydospores (@) 200300 / 400g SSF substrate). Surface sterilized (using $0.01 \% \mathrm{HgCl}_{2}$ ) sorghum seeds (@4 seeds / pot) were sown and grown with regular 
watering in glass house (day temperature maintained up-to $35+5^{0} \mathrm{C}$ ) for 30 days to prepare NI. The aerial plant parts were removed and the substrate was air dried with root pieces under shade for 24 hours.

\section{Production of Mass Inoculum (MI)}

In the field experiment, $25 \mathrm{~m}^{2}$ area was selected at the Instructional Research Farm, Department of Plant Pathology and Agricultural Microbiology, Mahatma Phule Krishi Vidyapeeth, Rahuri and well prepared. The whole area was divided into twenty plots with irrigation channels. Twenty plots represented twenty different treatment combinations. Three trap crop cycles of various host plants were cultivated for mass production technology of AM fungi.

\section{Experimental details}

\section{Selection of trap host plant}

The most important factor in choosing a host plant is selecting a plant that supports mycorrhizal growth. Crops such as spinach, sugar beet, lupine and members of mustard family do not form a symbiosis with AM fungi. Mainly monocots and plants with extensive root system are very good hosts for propagation of AM fungi. The five different trap crops viz., maize (Zea mays), fenugreek (Trigonella foenum-graecum), sorghum (Sorghum bicolor), pearl millet (Pennisetum typhoides L.) and coriander (Corriandrum sativum) were selected as host crops.

Soil solarization was done with LDPE mulching transparent sheet (25 micron) for 30 days (06 November 2017 to 06 December 2017).

The traps crops were sown randomly in twenty plots. Experiments were repeated three times in successive cycles. Each cycle had 30 days. After 30 days, the trap crops were cut at the base (topping). Sampling was done for observations. Second cycle sowing was started immediately after topping of first trap cycle crop. Locations of trap crops were changed. Similarly after 30 days, trap crops of second cycle were cut at the base and third round cycle sowing was done. Sampling was done after each production cycle.

\section{Sowing of trap crops}

Seeds of trap crops were sown according to their agronomic requirements. The sowing date of trap crop cycles were as follows,

\begin{tabular}{|l|l|}
\hline Trap crop cycle-I & 06 December 2017 \\
\hline Trap crop cycle-II & 04 January 2018 \\
\hline Trap crop cycle-III & 02 February 2018 \\
\hline
\end{tabular}

\section{Irrigation}

Irrigation schedule was followed as and when required for trap crops.

\section{Results and Discussion}

\section{On-farm production technology}

\section{Preparation of Nucleus Inoculum (NI)}

NI of native AMF (consortium) was prepared by the following method. Surface sterilized (used 'Chloramin $\mathrm{T}$ ' for 1 minute) chlamydospores of native AMF consortium, predominated by Glomus spp., Aculospora spp., Sceutellospora spp., Rhizophagus spp. and Gigaspora albida isolated from the natural root zone soils of eleven fruit crops from central campus MPKV, Rahuri by wet sieving and decanting method were multiplied on Sorghum (host plant) roots grown in 40 plastic pots filled with sterilized soil : sand : FYM mixture (1:1:1; v:v:v;) (SSF substrate) inoculated with native AMF chlamydospores 
(@) 200-300 / 400g SSF substrate) and sown with surface sterilized (using $0.01 \% \mathrm{HgCl}_{2}$ ) sorghum seeds (@ 4 seeds / pot), with regular watering in glass house (day temperature maintained up-to $35+5^{0} \mathrm{C}$ ) for 30 days to prepare NI. After the removal of aerial plant parts, air drying of substrate with root pieces was done under shade for 24 hours.

\section{Production of Mass Inoculum (MI)}

A field experiment for on-farm production of AM fungi was carried out at the Instructional Farm, Department of Plant Pathology and Agricultural Microbiology, Post Graduate Institute, Mahatma Phule Krishi Vidyapeeth, Rahuri.

The trap crops as host crop were sown randomly consisting of 20 treatments represented by five different trap crops viz., maize (Zea mays), fenugreek (Trigonella foenum-graecum), sorghum (Sorghum bicolor), pearl millet (Pennisetum typhoides L.) and coriander (Corriandrum sativum). Each treatment was grown in three successive trap crop cycle for 21 days duration (Plate 6). Each treatment was inoculated with Nucleus inoculum. The observations on AM fungal root colonization, number of spores and number of infective propagules were recorded after each trap cycle. These results are presented in the following tables.

\section{Trap crop cycle I}

\section{AM fungi percent root colonization}

The results from the above study showed that the treatment no. T16 (sorghum) (Table 1.1) had maximum per cent root colonization $(46.33 \%)$ followed by the treatment no. T3 (fenugreek) $(45.33 \%)$ and treatment no. T17 (fenugreek) $(44.00 \%)$. The treatment no. T2 (sorghum) showed the least per cent root colonization $(21.33 \%$ ) (Fig. 1).
Number of AM fungal spores $\left(100 \mathrm{~g}^{-1}\right)$ of root zone soils

The maximum number of AM fungal spores per $100 \mathrm{~g}$ of soil was observed in treatment $\mathrm{T}_{17}$ (fenugreek) (323.00) (Table 1.2) followed by the treatment $\mathrm{T}_{4}$ (maize) (321.66) and treatment $\mathrm{T}_{18}$ (maize) (317.00) whereas least number of AM fungal spores per $100 \mathrm{~g}$ of soil was observed treatment $\mathrm{T}_{1}$ (pearl millet) (225.33) (Fig. 2).

Infective propagules $\left(100 \mathrm{~g}^{-1}\right)$ of the root zone soils

The infective propagules (IP) per $100 \mathrm{~g}$ of soil showed that treatment $\mathrm{T}_{17}$ (fenugreek) had maximum IP 559.66 (Table 1.3) followed by treatment $\mathrm{T}_{15}$ (fenugreek) 555.66 and treatment $T_{3}$ (fenugreek) 552.33. The lowest value was recorded in treatment $\mathrm{T}_{20}$ (pearl millet) 392.33 (Fig. 3).

\section{Trap crop cycle II}

\section{AM fungi root colonization (\%)}

The per cent root colonization of AM fungi showed highest in treatment $\mathrm{T}_{3}$ (fenugreeksorghum) 56.33 per cent followed by treatment $\mathrm{T}_{4}$ (maize-pearl millet) 56.33 per cent and treatment $\mathrm{T}_{17}$ (fenugreek- maize) 55.00 per cent (Table 2.1). The lowest per cent root colonization was observed in treatment $\mathrm{T}_{20}$ (pearl millet- sorghum) 33.66 per cent (Fig. 4).

\section{Number of spores $\left(100 \mathrm{~g}^{-1}\right)$ of root zone soils}

The number of AM fungal spores (per $100 \mathrm{~g}$ of soil) in TC-II were recorded in treatment $\mathrm{T}_{17}$ (fenugreek - maize) 523.00 followed by treatment $\mathrm{T}_{11}$ (maize- fenugreek) 533.66 and treatment $\mathrm{T}_{4}$ (maize -pearl millet) 513.33 (Table 2.2). Lowest number of AM fungal spores (per $100 \mathrm{~g}$ of soil) was recorded in 
treatment $\mathrm{T}_{1}$ (pearl millet- fenugreek) 407.33 (Fig. 5).

\section{Infective propagules $\left(100 \mathrm{~g}^{-1}\right)$ of root zone soils}

After the second trap crop cycle treatment $\mathrm{T}_{15}$ (Fenugreek-Coriander) recorded the maximum IP count 577.33 per $100 \mathrm{~g}$ of soil followed by treatment $\mathrm{T}_{17}$ (Fenugreek- Maize) 572.33 per $100 \mathrm{~g}$ of soil (Table 2.3) while the minimum count was recorded in treatment $\mathrm{T}_{20}$ (Pearl Millet- Sorghum) 409.00 per $100 \mathrm{~g}$ of soil. 5.1.3 (Fig. 6).

\section{Trap crop cycle III}

\section{AM fungi root colonization (\%)}

The results in general indicated that after inoculation with nucleus inoculum, there was significant increase in the per cent root colonization in successive trap crop cycles. The highest percentage of root colonization was observed in treatment $\mathrm{T}_{4}$ (Maize - Pearl millet - Sorghum) 62.66 per cent and lowest colonization was recorded in treatment $\mathrm{T}_{7}$ (Maize-Sorghum- Pearl Millet) 47.33 per cent (Table 3.1).

The treatment $\mathrm{T}_{17}$ (fenugreek - maize sorghum) showed 59.66 per cent root colonization (Fig. 7). There was significant difference in root colonization percentage among the treatments. Maiti et al., (2009) multiplied MI on sorghum roots grown in partially sterilized soils of micro-plots $(4 \mathrm{x}$ $4 \mathrm{~m}^{2}$ ) and harvested 1775-2000 kg of MI.

Verma (2011) found berseem-berseemmarigold successive cropping gave the highest AM colonization followed by SorghumMethi-Berseem and Sorghum-MarigoldBerseem.

Mass production of AM fungi depends upon the type of host as well as duration of infection of these symbiotic organisms. Kadian et al., (2018) performed a pot experiment under polyhouse conditions, to evaluate the effect of chickpea husk as substrate with jowar (Sorghum bicolor), barley (Hordeum vulgare) and wheat (Triticum aestivum) as different host plant on mass multiplication of AM fungi. Among all the host plants, sorghum showed prominent results pertaining to maximum inoculum production of G. mosseae.

\section{Number of spores $\left(100 \mathrm{~g}^{-1}\right)$ of root zone soils}

The traps crops inoculated with nucleus inoculum produced significant number of spores for each trap crop cycle. The treatment $\mathrm{T}_{17}$ (fenugreek -maize-sorghum) showed highest spore count 693.66 (Table 3.2) whereas the lowest value was recorded in treatment $\mathrm{T}_{9}$ (pearl millet-corianderfenugreek) 444.33 (Fig. 8).

Verma (2011) found methi-berseem-maize the best combination among the twenty treatments, showing the highest average spore count per gram of soil.

Infective propagules $\left(100 \mathrm{~g}^{-1}\right)$ of root zone soils

Infective propagules of AM fungi include spores, fungal hyphae, extra radical mycelium, vesicles and arbuscules which are able to initiate new infection. The IP number per 100 gram of rhizosphere soil recorded shows significant differences among all the treatments. The highest value recorded was 620.33 in treatment $T_{17}$ (fenugreek - maize sorghum) and lowest observed was 354.66 in treatment $\mathrm{T}_{2}$ (sorghum- coriander- fenugreek) (Table 3.3 and Fig. 9). Comparisons of results of MPKV bioassays and spore populations indicated that vast majority of propagules are in the form other than spores, i.e., extraradical hyphae and colonized root pieces. 
Table.1.1 Root colonization (\%) of AM fungi in the root samples of selected trap crops

\begin{tabular}{|c|c|c|c|}
\hline $\begin{array}{l}\text { Treatment } \\
\text { No. }\end{array}$ & Trap Crop & $\begin{array}{c}\text { Root Colonization } \\
(\%)\end{array}$ & $\begin{array}{r}\text { Type of AM } \\
\text { Colonization }\end{array}$ \\
\hline $\mathbf{T}_{1}$ & Pearl Millet & 23.33 & $\mathbf{H}, \mathbf{V}, \mathbf{A}$ \\
\hline $\mathbf{T}_{2}$ & Sorghum & 21.33 & H \\
\hline $\mathbf{T}_{3}$ & Fenugreek & 45.33 & H,A \\
\hline $\mathbf{T}_{4}$ & Maize & 35.33 & $\mathrm{H}, \mathrm{V}$ \\
\hline $\mathbf{T}_{5}$ & Coriander & 37.33 & $\mathrm{H}, \mathrm{V}$ \\
\hline $\mathbf{T}_{6}$ & Fenugreek & 25.66 & H,A \\
\hline $\mathbf{T}_{7}$ & Maize & 27.33 & $\mathbf{H}, \mathbf{V}, \mathbf{A}$ \\
\hline $\mathbf{T}_{8}$ & Coriander & 33.33 & $\mathbf{H}, \mathbf{V}, \mathbf{A}$ \\
\hline$T_{9}$ & Pearl Millet & 26.33 & $\mathbf{H}, \mathbf{V}, \mathbf{A}$ \\
\hline $\mathbf{T}_{10}$ & Sorghum & 41.66 & $\mathrm{H}, \mathrm{V}$ \\
\hline$T_{11}$ & Maize & 27.00 & H,A \\
\hline $\mathbf{T}_{12}$ & Coriander & 23.00 & $\mathrm{H}, \mathrm{V}$ \\
\hline $\mathbf{T}_{13}$ & Pearl Millet & 35.00 & H,A \\
\hline$T_{14}$ & Sorghum & 36.33 & H,A \\
\hline $\mathbf{T}_{15}$ & Fenugreek & 40.00 & $\mathbf{H}, \mathbf{V}, \mathbf{A}$ \\
\hline $\mathbf{T}_{16}$ & Sorghum & 46.33 & $\mathbf{H}, \mathbf{V}, \mathbf{A}$ \\
\hline$T_{17}$ & Fenugreek & 44.00 & H,A \\
\hline $\mathbf{T}_{18}$ & Maize & 33.33 & H,A \\
\hline$T_{19}$ & Coriander & 22.00 & $\mathrm{H}, \mathrm{V}$ \\
\hline \multirow[t]{4}{*}{$\mathbf{T}_{20}$} & Pearl Millet & 38.66 & H,A \\
\hline & S.E. \pm & 2.18 & \\
\hline & CD at $5 \%$ & 6.23 & \\
\hline & CD at $1 \%$ & 8.34 & \\
\hline
\end{tabular}

H-Hyphal colonization, V-Vesicular colonization, A-Arbuscular colonization 
Table.1.2 Number of AM fungal spores $\left(100 \mathrm{~g}^{-1}\right)$ recovered from the root zone soils of selected trap crops

\begin{tabular}{|c|c|c|}
\hline $\begin{array}{l}\text { Treatment } \\
\text { No. }\end{array}$ & Trap Crop & $\begin{array}{l}\text { Number of AM fungal spores } \\
\left(100 \mathrm{~g}^{-1} \text { of soil }\right)\end{array}$ \\
\hline $\mathbf{T}_{1}$ & Pearl Millet & 225.33 \\
\hline $\mathbf{T}_{2}$ & Sorghum & 275.66 \\
\hline $\mathbf{T}_{\mathbf{3}}$ & Fenugreek & 296.00 \\
\hline $\mathbf{T}_{4}$ & Maize & 321.66 \\
\hline $\mathbf{T}_{5}$ & Coriander & 265.66 \\
\hline $\mathbf{T}_{6}$ & Fenugreek & 304.00 \\
\hline $\mathbf{T}_{7}$ & Maize & 312.00 \\
\hline $\mathbf{T}_{8}$ & Coriander & 259.66 \\
\hline $\mathbf{T}_{9}$ & Pearl Millet & 232.33 \\
\hline $\mathbf{T}_{10}$ & Sorghum & 273.00 \\
\hline $\mathbf{T}_{11}$ & Maize & 312.66 \\
\hline $\mathbf{T}_{12}$ & Coriander & 265.00 \\
\hline $\mathbf{T}_{13}$ & Pearl Millet & 220.33 \\
\hline $\mathbf{T}_{14}$ & Sorghum & 278.33 \\
\hline $\mathbf{T}_{15}$ & Fenugreek & 301.66 \\
\hline $\mathbf{T}_{16}$ & Sorghum & 283.00 \\
\hline $\mathbf{T}_{17}$ & Fenugreek & 323.00 \\
\hline $\mathbf{T}_{18}$ & Maize & 317.00 \\
\hline $\mathbf{T}_{19}$ & Coriander & 263.00 \\
\hline \multirow[t]{4}{*}{$\mathbf{T}_{\mathbf{2 0}}$} & Pearl Millet & 227.00 \\
\hline & S.E. \pm & 1.98 \\
\hline & CD at $5 \%$ & 5.66 \\
\hline & CD at $1 \%$ & 7.57 \\
\hline
\end{tabular}


Table.1.3 Infective propagules $\left(100 \mathrm{~g}^{-1}\right)$ recovered from root zone soils of selected trap crops

\begin{tabular}{|c|c|c|}
\hline $\begin{array}{l}\text { Treatment } \\
\text { No. }\end{array}$ & Trap Crop & $\begin{array}{c}\text { Infectious propagules (IP) } \\
\left(100 \mathrm{~g}^{-1} \text { of soil }\right)\end{array}$ \\
\hline $\mathbf{T}_{1}$ & Pearl Millet & 408.66 \\
\hline $\mathbf{T}_{2}$ & Sorghum & 496.00 \\
\hline $\mathbf{T}_{\mathbf{3}}$ & Fenugreek & 552.33 \\
\hline $\mathbf{T}_{4}$ & Maize & 507.33 \\
\hline $\mathbf{T}_{5}$ & Coriander & 478.33 \\
\hline $\mathbf{T}_{6}$ & Fenugreek & 550.00 \\
\hline $\mathbf{T}_{7}$ & Maize & 510.00 \\
\hline $\mathbf{T}_{8}$ & Coriander & 482.00 \\
\hline $\mathbf{T}_{9}$ & Pearl Millet & 410.00 \\
\hline$T_{10}$ & Sorghum & $\mathbf{5 0 7 . 3 3}$ \\
\hline$T_{11}$ & Maize & 509.00 \\
\hline $\mathbf{T}_{12}$ & Coriander & 478.33 \\
\hline $\mathbf{T}_{13}$ & Pearl Millet & 397.66 \\
\hline$T_{14}$ & Sorghum & 494.66 \\
\hline$T_{15}$ & Fenugreek & 555.66 \\
\hline $\mathbf{T}_{16}$ & Sorghum & 494.66 \\
\hline $\mathbf{T}_{17}$ & Fenugreek & 559.66 \\
\hline $\mathbf{T}_{18}$ & Maize & $\mathbf{5 0 7 . 3 3}$ \\
\hline$T_{19}$ & Coriander & 474.66 \\
\hline \multirow[t]{4}{*}{$\mathbf{T}_{20}$} & Pearl Millet & 392.33 \\
\hline & S.E. \pm & 1.89 \\
\hline & CD at $5 \%$ & 5.40 \\
\hline & CD at $1 \%$ & 7.23 \\
\hline
\end{tabular}


Table.2.1 Root colonization (\%) of AM fungi in the root samples of selected trap crops

\begin{tabular}{|c|c|c|c|c|}
\hline $\begin{array}{c}\text { Treatment } \\
\text { No. }\end{array}$ & $\begin{array}{c}\text { Previous Crop } \\
\text { (TCC-I) }\end{array}$ & Trap Crop & $\begin{array}{c}\text { Root } \\
\text { Colonization }(\%)\end{array}$ & $\begin{array}{l}\text { Type of AM } \\
\text { Colonization }\end{array}$ \\
\hline $\mathbf{T}_{1}$ & Pearl Millet & Fenugreek & 34.66 & $\mathbf{H}, \mathbf{V}, \mathbf{A}$ \\
\hline $\mathbf{T}_{2}$ & Sorghum & Coriander & 35.00 & H,A \\
\hline $\mathbf{T}_{3}$ & Fenugreek & Sorghum & 56.33 & H,A \\
\hline $\mathbf{T}_{4}$ & Maize & Pearl Millet & 56.00 & $\mathbf{H}, \mathbf{V}$ \\
\hline $\mathbf{T}_{5}$ & Coriander & Maize & 44.33 & $\mathbf{H}, \mathbf{V}$ \\
\hline $\mathbf{T}_{6}$ & Fenugreek & Pearl Millet & 44.66 & H,A \\
\hline $\mathbf{T}_{7}$ & Maize & Sorghum & 35.33 & $\mathbf{H}, \mathbf{V}, \mathbf{A}$ \\
\hline $\mathbf{T}_{8}$ & Coriander & Fenugreek & 45.66 & $\mathbf{H}, \mathbf{V}, \mathbf{A}$ \\
\hline $\mathbf{T}_{9}$ & Pearl Millet & Coriander & 34.66 & $\mathbf{H}, \mathbf{V}, \mathbf{A}$ \\
\hline $\mathbf{T}_{10}$ & Sorghum & Maize & 50.33 & $\mathbf{H}, \mathbf{V}, \mathbf{A}$ \\
\hline $\mathbf{T}_{11}$ & Maize & Fenugreek & 35.33 & H,A \\
\hline $\mathbf{T}_{12}$ & Coriander & Sorghum & 45.66 & H,V \\
\hline $\mathbf{T}_{13}$ & Pearl Millet & Maize & 38.66 & H,A \\
\hline $\mathbf{T}_{14}$ & Sorghum & Perl Millet & 37.33 & H,A \\
\hline $\mathbf{T}_{15}$ & Fenugreek & Coriander & 43.66 & $\mathbf{H}, \mathbf{V}, \mathbf{A}$ \\
\hline $\mathbf{T}_{16}$ & Sorghum & Fenugreek & 54.33 & $\mathbf{H}, \mathbf{V}, \mathbf{A}$ \\
\hline $\mathbf{T}_{17}$ & Fenugreek & Maize & 55.00 & H,A \\
\hline $\mathbf{T}_{18}$ & Maize & Coriander & 48.66 & H,A \\
\hline $\mathbf{T}_{19}$ & Coriander & Pearl Millet & 46.00 & $\mathbf{H}, \mathbf{V}$ \\
\hline \multirow[t]{4}{*}{$\mathbf{T}_{20}$} & Pearl Millet & Sorghum & 33.66 & $\mathbf{H}, \mathbf{V}, \mathbf{A}$ \\
\hline & S.E. \pm & & 1.77 & \\
\hline & CD at $5 \%$ & & 5.07 & \\
\hline & CD at $1 \%$ & & 6.79 & \\
\hline
\end{tabular}

H-Hyphal colonization, V-Vesicular colonization, A-Arbuscular colonization 
Table.2.2 Number of AM fungal spores $\left(100 \mathrm{~g}^{-1}\right)$ recovered from root zone soils of selected trap crops

\begin{tabular}{|c|c|c|c|}
\hline $\begin{array}{l}\text { Treatment } \\
\text { No. }\end{array}$ & $\begin{array}{l}\text { Previous Crop } \\
\text { (TCC-I) }\end{array}$ & Trap Crop & $\begin{array}{l}\text { Number of AM fungal } \\
\text { spores ( } 100 \mathrm{~g}^{-1} \text { of soil) }\end{array}$ \\
\hline $\mathbf{T}_{1}$ & Pearl Millet & Fenugreek & 407.33 \\
\hline $\mathbf{T}_{2}$ & Sorghum & Coriander & 464.66 \\
\hline $\mathbf{T}_{3}$ & Fenugreek & Sorghum & 490.00 \\
\hline $\mathbf{T}_{4}$ & Maize & Pearl Millet & 513.33 \\
\hline $\mathbf{T}_{5}$ & Coriander & Maize & 453.00 \\
\hline $\mathbf{T}_{6}$ & Fenugreek & Pearl Millet & 492.00 \\
\hline $\mathbf{T}_{7}$ & Maize & Sorghum & 502.00 \\
\hline $\mathbf{T}_{8}$ & Coriander & Fenugreek & 426.66 \\
\hline $\mathbf{T}_{9}$ & Pearl Millet & Coriander & 413.00 \\
\hline$T_{10}$ & Sorghum & Maize & 475.33 \\
\hline$T_{11}$ & Maize & Fenugreek & 533.66 \\
\hline $\mathbf{T}_{12}$ & Coriander & Sorghum & 458.33 \\
\hline $\mathbf{T}_{13}$ & Pearl Millet & Maize & 422.33 \\
\hline $\mathbf{T}_{14}$ & Sorghum & Perl Millet & 475.33 \\
\hline$T_{15}$ & Fenugreek & Coriander & 509.66 \\
\hline$T_{16}$ & Sorghum & Fenugreek & 463.33 \\
\hline$T_{17}$ & Fenugreek & Maize & 523.00 \\
\hline $\mathbf{T}_{18}$ & Maize & Coriander & 512.33 \\
\hline $\mathbf{T}_{19}$ & Coriander & Pearl Millet & 454.00 \\
\hline \multirow[t]{4}{*}{$T_{20}$} & Pearl Millet & Sorghum & 426.00 \\
\hline & S.E. \pm & & 1.49 \\
\hline & CD at $5 \%$ & & 4.27 \\
\hline & CD at $1 \%$ & & 5.71 \\
\hline
\end{tabular}


Table.2.3 Infective propagules $\left(100 \mathrm{~g}^{-1}\right)$ recovered from root zone soils of selected trap crops

\begin{tabular}{|c|c|c|c|}
\hline $\begin{array}{l}\text { Treatment } \\
\text { No. }\end{array}$ & $\begin{array}{l}\text { Previous Crop } \\
\text { (TCC-I) }\end{array}$ & Trap Crop & $\begin{array}{c}\text { Infective propagules (IP) } \\
\left(100 \mathrm{~g}^{-1} \text { of soil }\right)\end{array}$ \\
\hline $\mathbf{T}_{1}$ & Pearl Millet & Fenugreek & 431.33 \\
\hline $\mathbf{T}_{2}$ & Sorghum & Coriander & 509.66 \\
\hline $\mathbf{T}_{3}$ & Fenugreek & Sorghum & 566.33 \\
\hline $\mathbf{T}_{4}$ & Maize & Pearl Millet & 514.66 \\
\hline $\mathbf{T}_{5}$ & Coriander & Maize & 497.00 \\
\hline$T_{6}$ & Fenugreek & Pearl Millet & 557.00 \\
\hline $\mathbf{T}_{7}$ & Maize & Sorghum & 523.00 \\
\hline $\mathbf{T}_{8}$ & Coriander & Fenugreek & 495.66 \\
\hline $\mathbf{T}_{9}$ & Pearl Millet & Coriander & 422.66 \\
\hline$T_{10}$ & Sorghum & Maize & 523.33 \\
\hline$T_{11}$ & Maize & Fenugreek & 536.00 \\
\hline$T_{12}$ & Coriander & Sorghum & 492.66 \\
\hline$T_{13}$ & Pearl Millet & Maize & 422.66 \\
\hline $\mathbf{T}_{14}$ & Sorghum & Perl Millet & 511.33 \\
\hline $\mathbf{T}_{15}$ & Fenugreek & Coriander & 577.33 \\
\hline$T_{16}$ & Sorghum & Fenugreek & 514.66 \\
\hline $\mathbf{T}_{17}$ & Fenugreek & Maize & 572.33 \\
\hline $\mathbf{T}_{18}$ & Maize & Coriander & 524.00 \\
\hline$T_{19}$ & Coriander & Pearl Millet & 484.33 \\
\hline \multirow[t]{4}{*}{$\mathbf{T}_{20}$} & Pearl Millet & Sorghum & 409.00 \\
\hline & S.E. \pm & & 1.78 \\
\hline & CD at $5 \%$ & & 5.10 \\
\hline & CD at $1 \%$ & & 6.83 \\
\hline
\end{tabular}


Table.3.1 Root colonization (\%) of AM fungi in the root samples of selected trap crops

\begin{tabular}{|c|c|c|c|c|c|}
\hline $\begin{array}{c}\text { Treatment } \\
\text { No. }\end{array}$ & $\begin{array}{l}\text { Previous } \\
\text { Crop } \\
\text { (TCC-I) }\end{array}$ & $\begin{array}{l}\text { Previous } \\
\text { Crop } \\
\text { (TCC-II) }\end{array}$ & Trap Crop & $\begin{array}{c}\text { Root } \\
\text { Colonization } \\
(\%)\end{array}$ & $\begin{array}{l}\text { Type of AM } \\
\text { Colonization }\end{array}$ \\
\hline $\mathbf{T}_{1}$ & Pearl Millet & Fenugreek & Maize & 47.33 & $\mathbf{H}, \mathbf{V}, \mathbf{A}$ \\
\hline $\mathbf{T}_{2}$ & Sorghum & Coriander & Fenugreek & 45.66 & H,A \\
\hline $\mathbf{T}_{\mathbf{3}}$ & Fenugreek & Sorghum & Coriander & 58.33 & H,A \\
\hline $\mathbf{T}_{4}$ & Maize & Pearl Millet & Sorghum & 62.66 & $\mathbf{H}, \mathbf{V}$ \\
\hline $\mathbf{T}_{5}$ & Coriander & Maize & Pearl Millet & 54.66 & H, V,A \\
\hline $\mathbf{T}_{6}$ & Fenugreek & Pearl Millet & Sorghum & 55.00 & H,A \\
\hline $\mathbf{T}_{7}$ & Maize & Sorghum & Pearl Millet & 45.33 & $\mathbf{H}, \mathbf{V}, \mathbf{A}$ \\
\hline $\mathbf{T}_{8}$ & Coriander & Fenugreek & Maize & 48.33 & $\mathbf{H}, \mathbf{V}, \mathbf{A}$ \\
\hline $\mathbf{T}_{9}$ & Pearl Millet & Coriander & Fenugreek & 47.00 & $\mathbf{H}, \mathbf{V}, \mathbf{A}$ \\
\hline $\mathbf{T}_{10}$ & Sorghum & Maize & Coriander & 58.33 & $\mathbf{H}, \mathbf{V}, \mathbf{A}$ \\
\hline $\mathbf{T}_{11}$ & Maize & Fenugreek & Coriander & 47.00 & H,A \\
\hline $\mathbf{T}_{12}$ & Coriander & Sorghum & Pearl Millet & 58.00 & H, V,A \\
\hline $\mathbf{T}_{13}$ & Pearl Millet & Maize & Sorghum & 47.66 & H,A \\
\hline $\mathbf{T}_{14}$ & Sorghum & Perl Millet & Fenugreek & 53.66 & H,A \\
\hline $\mathbf{T}_{15}$ & Fenugreek & Coriander & Maize & 56.00 & $\mathbf{H}, \mathbf{V}, \mathbf{A}$ \\
\hline $\mathbf{T}_{16}$ & Sorghum & Fenugreek & Pearl Millet & 59.66 & $\mathbf{H}, \mathbf{V}, \mathbf{A}$ \\
\hline $\mathbf{T}_{17}$ & Fenugreek & Maize & Sorghum & 59.66 & H,A \\
\hline $\mathbf{T}_{18}$ & Maize & Coriander & Fenugreek & 53.33 & $\mathbf{H}, \mathbf{V}, \mathbf{A}$ \\
\hline $\mathbf{T}_{19}$ & Coriander & Pearl Millet & Maize & 55.66 & $\mathbf{H}, \mathbf{V}$ \\
\hline \multirow[t]{4}{*}{$\mathbf{T}_{20}$} & Pearl Millet & Sorghum & Coriander & 59.00 & $\mathbf{H}, \mathbf{V}, \mathbf{A}$ \\
\hline & S.E. \pm & & & 1.85 & \\
\hline & CD at $5 \%$ & & & 5.30 & \\
\hline & CD at $1 \%$ & & & 7.09 & \\
\hline
\end{tabular}

H-Hyphal colonization, V-Vesicular colonization, A-Arbuscular colonization 
Table.3.2 Number of AM fungal spores $\left(100 \mathrm{~g}^{-1}\right)$ recovered from root zone soils of selected trap crops

\begin{tabular}{|c|l|l|l|c|}
\hline $\begin{array}{c}\text { Treatment } \\
\text { No. }\end{array}$ & $\begin{array}{c}\text { Previous Crop } \\
\text { (TCC-I) }\end{array}$ & $\begin{array}{c}\text { Previous Crop } \\
\text { (TCC-II) }\end{array}$ & Trap Crop & $\begin{array}{c}\text { Average number of } \\
\text { AM fungal spores } \\
\left(\mathbf{1 0 0} \mathbf{g}^{-1} \text { of soil) }\right.\end{array}$ \\
\hline $\mathbf{T}_{\mathbf{1}}$ & Pearl Millet & Fenugreek & Maize & $\mathbf{5 2 0 . 6 6}$ \\
\hline $\mathbf{T}_{\mathbf{2}}$ & Sorghum & Coriander & Fenugreek & $\mathbf{5 5 4 . 0 0}$ \\
\hline $\mathbf{T}_{\mathbf{3}}$ & Fenugreek & Sorghum & Coriander & $\mathbf{6 5 3 . 0 0}$ \\
\hline $\mathbf{T}_{\mathbf{4}}$ & Maize & Pearl Millet & Sorghum & $\mathbf{5 6 2 . 6 6}$ \\
\hline $\mathbf{T}_{\mathbf{5}}$ & Coriander & Maize & Pearl Millet & $\mathbf{5 2 4 . 3 3}$ \\
\hline $\mathbf{T}_{\mathbf{6}}$ & Fenugreek & Pearl Millet & Sorghum & $\mathbf{5 0 1 . 3 3}$ \\
\hline $\mathbf{T}_{\mathbf{7}}$ & Maize & Sorghum & Pearl Millet & $\mathbf{6 0 9 . 0 0}$ \\
\hline $\mathbf{T}_{\mathbf{8}}$ & Coriander & Fenugreek & Maize & $\mathbf{4 9 7 . 6 6}$ \\
\hline $\mathbf{T}_{\mathbf{9}}$ & Pearl Millet & Coriander & Fenugreek & $\mathbf{4 4 4 . 3 3}$ \\
\hline $\mathbf{T}_{\mathbf{1 0}}$ & Sorghum & Maize & Coriander & $\mathbf{5 0 1 . 6 6}$ \\
\hline $\mathbf{T}_{\mathbf{1 1}}$ & Maize & Fenugreek & Coriander & $\mathbf{5 9 4 . 0 0}$ \\
\hline $\mathbf{T}_{\mathbf{1 2}}$ & Coriander & Sorghum & Pearl Millet & $\mathbf{4 8 0 . 3 3}$ \\
\hline $\mathbf{T}_{\mathbf{1 3}}$ & Pearl Millet & Maize & Sorghum & $\mathbf{4 6 6 . 3 3}$ \\
\hline $\mathbf{T}_{\mathbf{1 4}}$ & Sorghum & Perl Millet & Fenugreek & $\mathbf{5 0 8 . 0 0}$ \\
\hline $\mathbf{T}_{\mathbf{1 5}}$ & Fenugreek & Coriander & Maize & $\mathbf{5 3 2 . 0 0}$ \\
\hline $\mathbf{T}_{\mathbf{1 6}}$ & Sorghum & Fenugreek & Pearl Millet & $\mathbf{4 9 3 . 6 6}$ \\
\hline $\mathbf{T}_{\mathbf{1 7}}$ & Fenugreek & Maize & Sorghum & $\mathbf{6 9 3 . 6 6}$ \\
\hline $\mathbf{T}_{\mathbf{1 8}}$ & Maize & Coriander & Fenugreek & $\mathbf{6 3 2 . 6 6}$ \\
\hline $\mathbf{T}_{\mathbf{1 9}}$ & Coriander & Pearl Millet & Maize & $\mathbf{4 9 1 . 0 0}$ \\
\hline $\mathbf{T}_{\mathbf{2 0}}$ & Pearl Millet & Sorghum & Coriander & $\mathbf{4 5 4 . 3 3}$ \\
\hline & S.E. \pm & & & $\mathbf{1 . 8 5}$ \\
\hline & CD at $\mathbf{5} \%$ & CD at $\mathbf{1} \%$ & & $\mathbf{5 . 3 0}$ \\
\hline & & & $\mathbf{7 . 0 9}$ \\
\hline & & & \\
\hline & & & \\
\hline
\end{tabular}


Table.3.3 Infective propagules $\left(100 \mathrm{~g}^{-1}\right)$ recovered from the root zone soils of selected trap crops

\begin{tabular}{|c|l|l|l|c|}
\hline $\begin{array}{c}\text { Treatment } \\
\text { No. }\end{array}$ & $\begin{array}{c}\text { Previous Crop } \\
\text { (TCC-I) }\end{array}$ & $\begin{array}{c}\text { Previous Crop } \\
\text { (TCC-II) }\end{array}$ & Trap Crop & $\begin{array}{c}\text { Infectious } \\
\text { propagules (IP) } \\
\left(\mathbf{1 0 0} \mathbf{g}^{-1} \text { of soil) }\right.\end{array}$ \\
\hline $\mathbf{T}_{\mathbf{1}}$ & Pearl Millet & Fenugreek & Maize & $\mathbf{4 6 2 . 3 3}$ \\
\hline $\mathbf{T}_{\mathbf{2}}$ & Sorghum & Coriander & Fenugreek & $\mathbf{3 5 4 . 6 6}$ \\
\hline $\mathbf{T}_{\mathbf{3}}$ & Fenugreek & Sorghum & Coriander & $\mathbf{5 4 7 . 3 3}$ \\
\hline $\mathbf{T}_{\mathbf{4}}$ & Maize & Pearl Millet & Sorghum & $\mathbf{5 8 8 . 0 0}$ \\
\hline $\mathbf{T}_{\mathbf{5}}$ & Coriander & Maize & Pearl Millet & $\mathbf{5 3 2 . 3 3}$ \\
\hline $\mathbf{T}_{\mathbf{6}}$ & Fenugreek & Pearl Millet & Sorghum & $\mathbf{5 5 7 . 3 3}$ \\
\hline $\mathbf{T}_{\mathbf{7}}$ & Maize & Sorghum & Pearl Millet & $\mathbf{5 8 5 . 0 0}$ \\
\hline $\mathbf{T}_{\mathbf{8}}$ & Coriander & Fenugreek & Maize & $\mathbf{5 2 7 . 6 6}$ \\
\hline $\mathbf{T}_{\mathbf{9}}$ & Pearl Millet & Coriander & Fenugreek & $\mathbf{4 4 5 . 6 6}$ \\
\hline $\mathbf{T}_{\mathbf{1 0}}$ & Sorghum & Maize & Coriander & $\mathbf{5 5 7 . 3 3}$ \\
\hline $\mathbf{T}_{\mathbf{1 1}}$ & Maize & Fenugreek & Coriander & $\mathbf{6 1 1 . 0 0}$ \\
\hline $\mathbf{T}_{\mathbf{1 2}}$ & Coriander & Sorghum & Pearl Millet & $\mathbf{5 2 5 . 3 3}$ \\
\hline $\mathbf{T}_{\mathbf{1 3}}$ & Pearl Millet & Maize & Sorghum & $\mathbf{4 5 7 . 6 6}$ \\
\hline $\mathbf{T}_{\mathbf{1 4}}$ & Sorghum & Perl Millet & Fenugreek & $\mathbf{5 4 4 . 3 3}$ \\
\hline $\mathbf{T}_{\mathbf{1 5}}$ & Fenugreek & Coriander & Maize & $\mathbf{5 7 2 . 0 0}$ \\
\hline $\mathbf{T}_{\mathbf{1 6}}$ & Sorghum & Fenugreek & Pearl Millet & $\mathbf{5 4 7 . 6 6}$ \\
\hline $\mathbf{T}_{\mathbf{1 7}}$ & Fenugreek & Maize & Sorghum & $\mathbf{6 2 0 . 3 3}$ \\
\hline $\mathbf{T}_{\mathbf{1 8}}$ & Maize & Coriander & Fenugreek & $\mathbf{5 5 9 . 6 6}$ \\
\hline $\mathbf{T}_{\mathbf{1 9}}$ & Coriander & Pearl Millet & Maize & $\mathbf{5 1 2 . 3 3}$ \\
\hline $\mathbf{T}_{\mathbf{2 0}}$ & Pearl Millet & Sorghum & Coriander & $\mathbf{4 5 7 . 0 0}$ \\
\hline & S.E. $\mathbf{\pm}$ & & & $\mathbf{3 . 6 5}$ \\
\hline & CD at $\mathbf{5} \%$ & & & $\mathbf{1 0 . 4 3}$ \\
\hline & CD at $\mathbf{1} \%$ & & & $\mathbf{1 3 . 9 6}$ \\
\hline & & & & \\
\hline
\end{tabular}




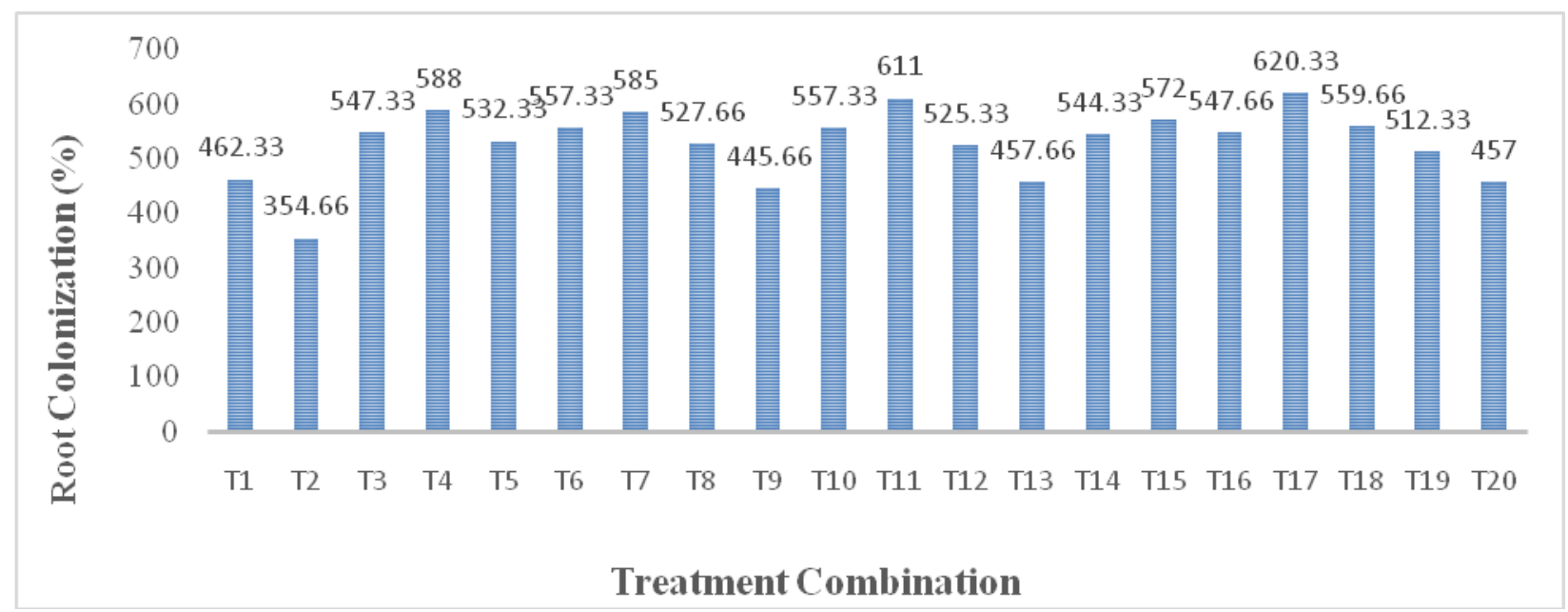

Fig.1 AM fungi root colonization (\%) in Trap Crop Cycle-I

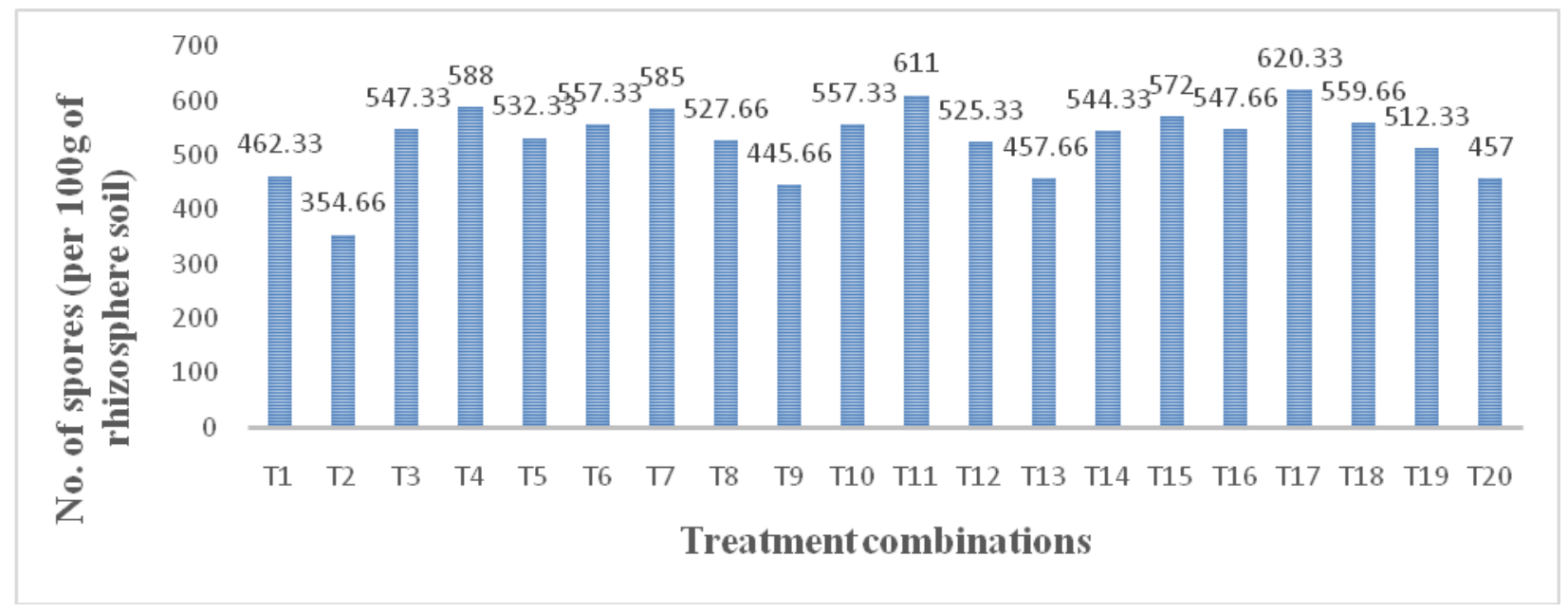

Fig.2 Number of spores per 100g of rhizosphere soil in Trap Crop Cycle-I

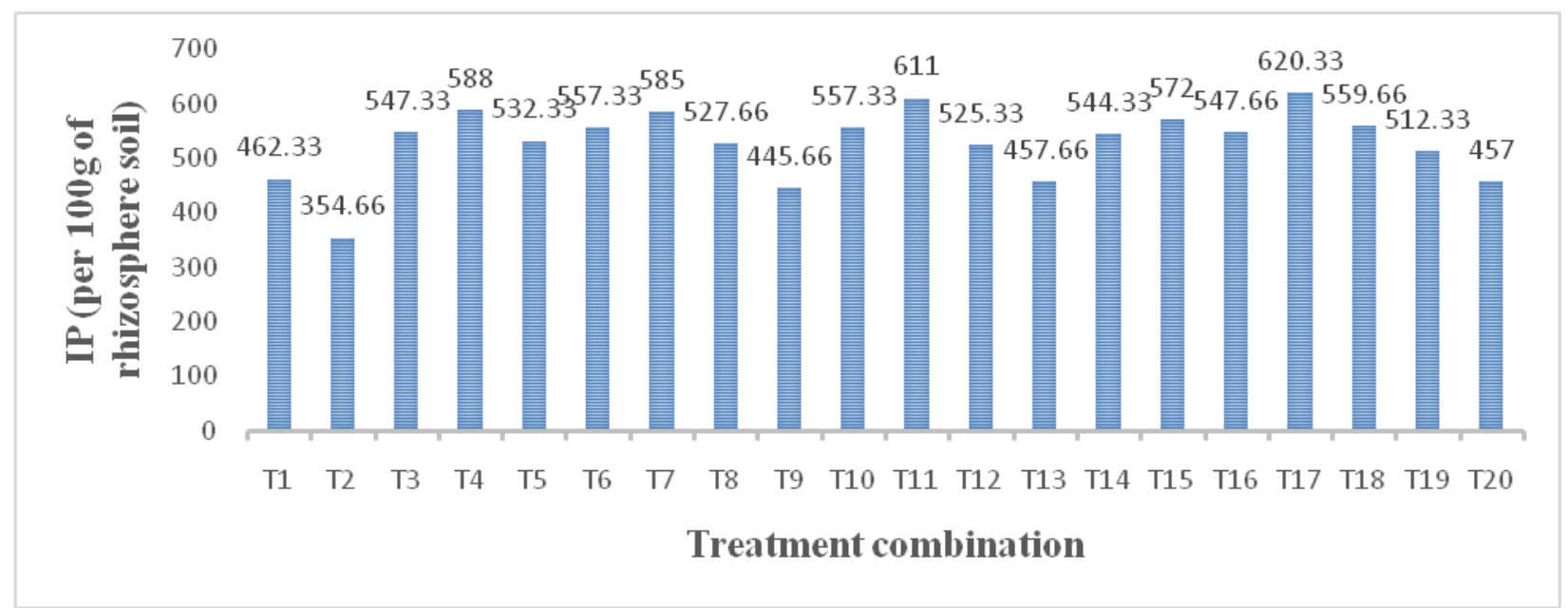

Fig.3 Infectious propagules of AMF per 100 gram of rhizosphere soil in Trap Crop Cycle-I 


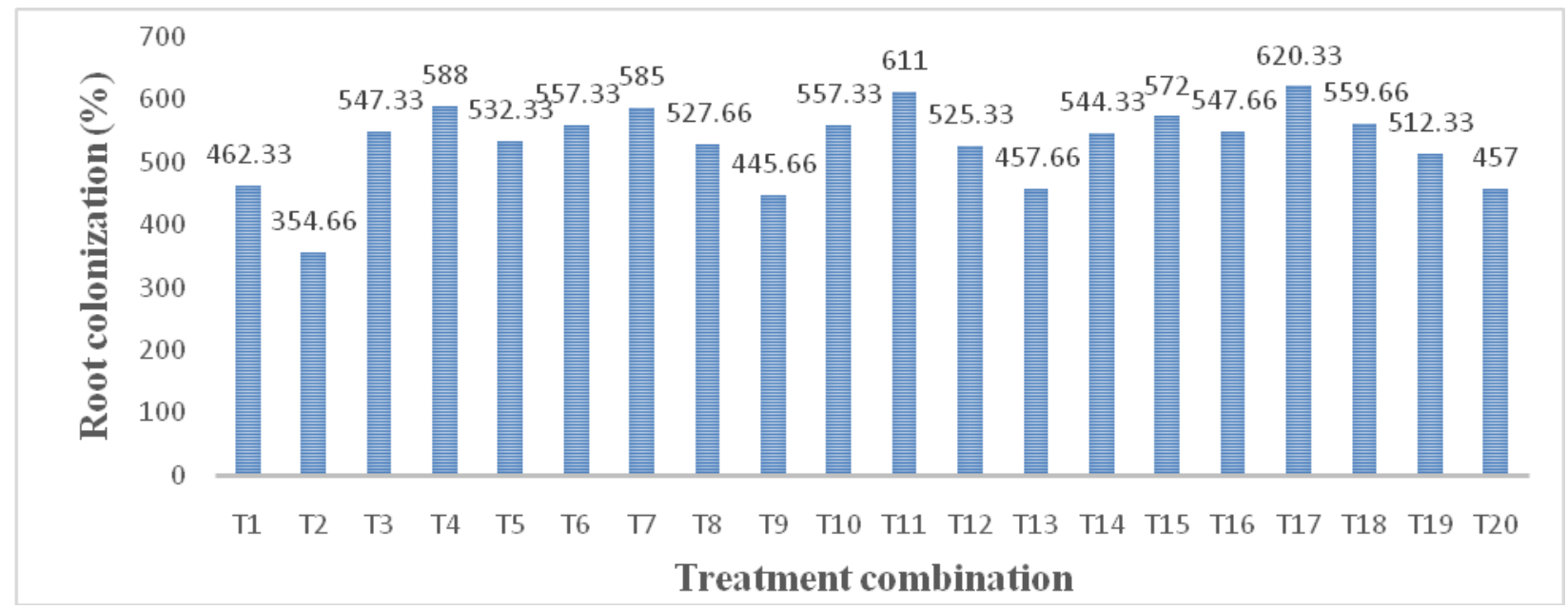

Fig.4 AM fungi root colonization (\%) in Trap Crop Cycle-II

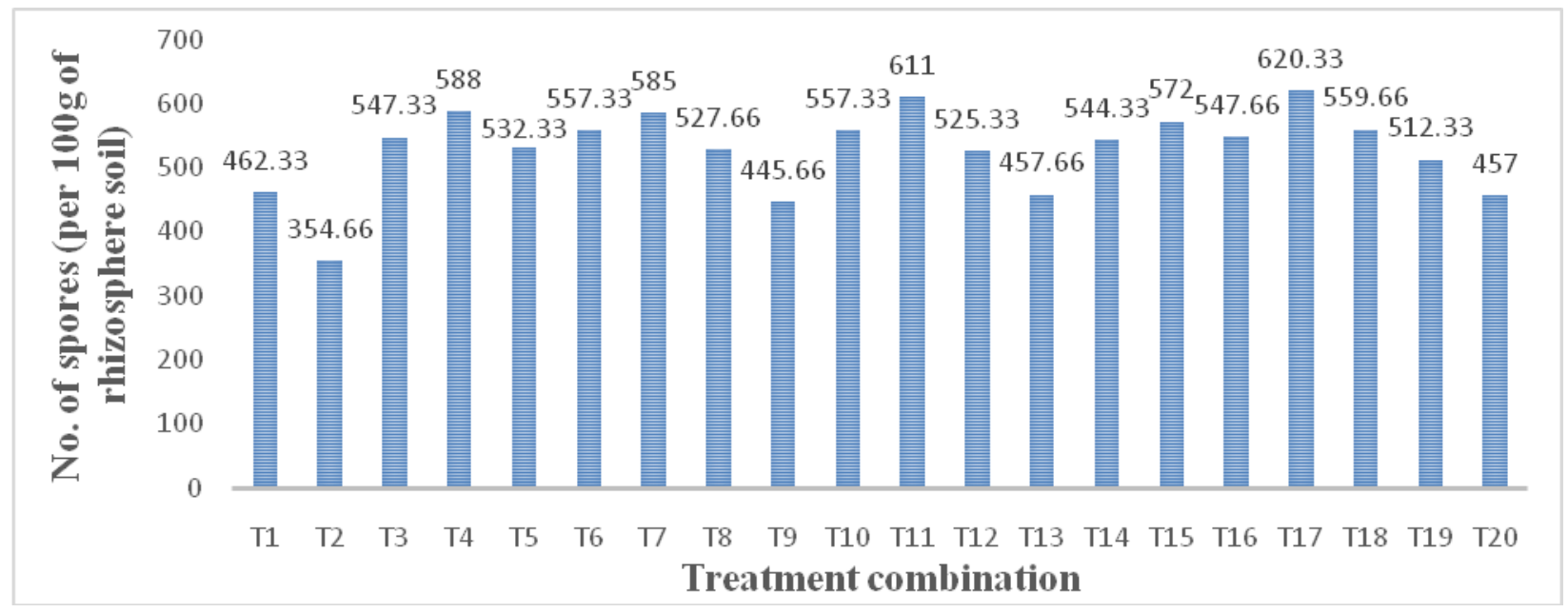

Fig.5 Number of spores per 100g of rhizosphere soil in Trap Crop Cycle-II

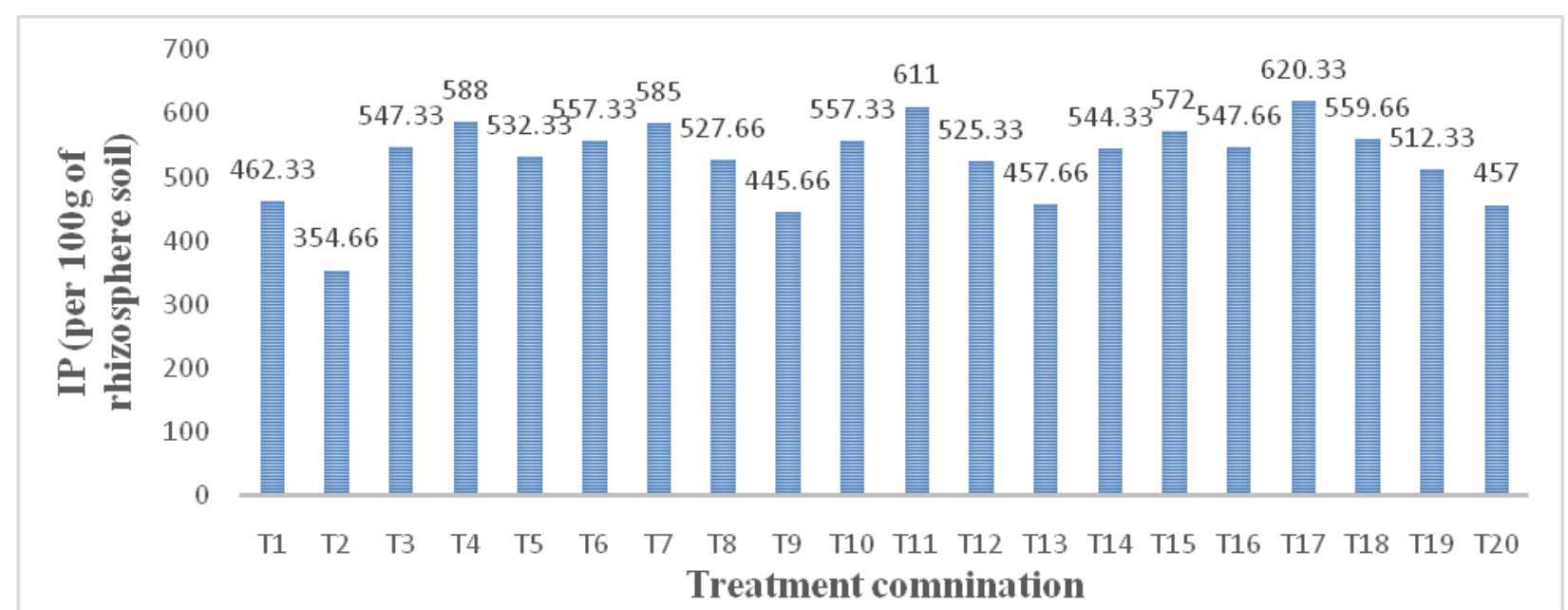

Fig.6 Infectious propagules of AMF per 100 gram of rhizosphere soil in Trap Crop Cycle-II 


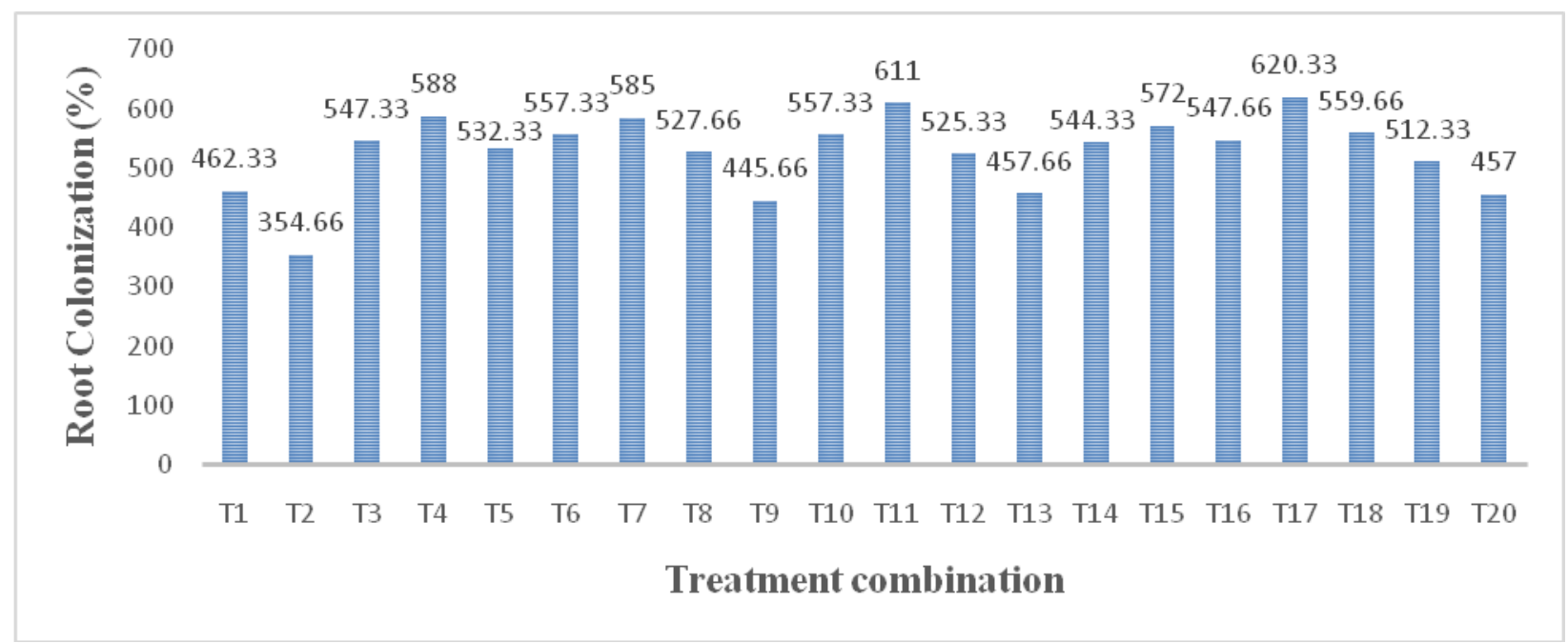

Fig.7 AM fungi root colonization (\%) in Trap Crop Cycle-III

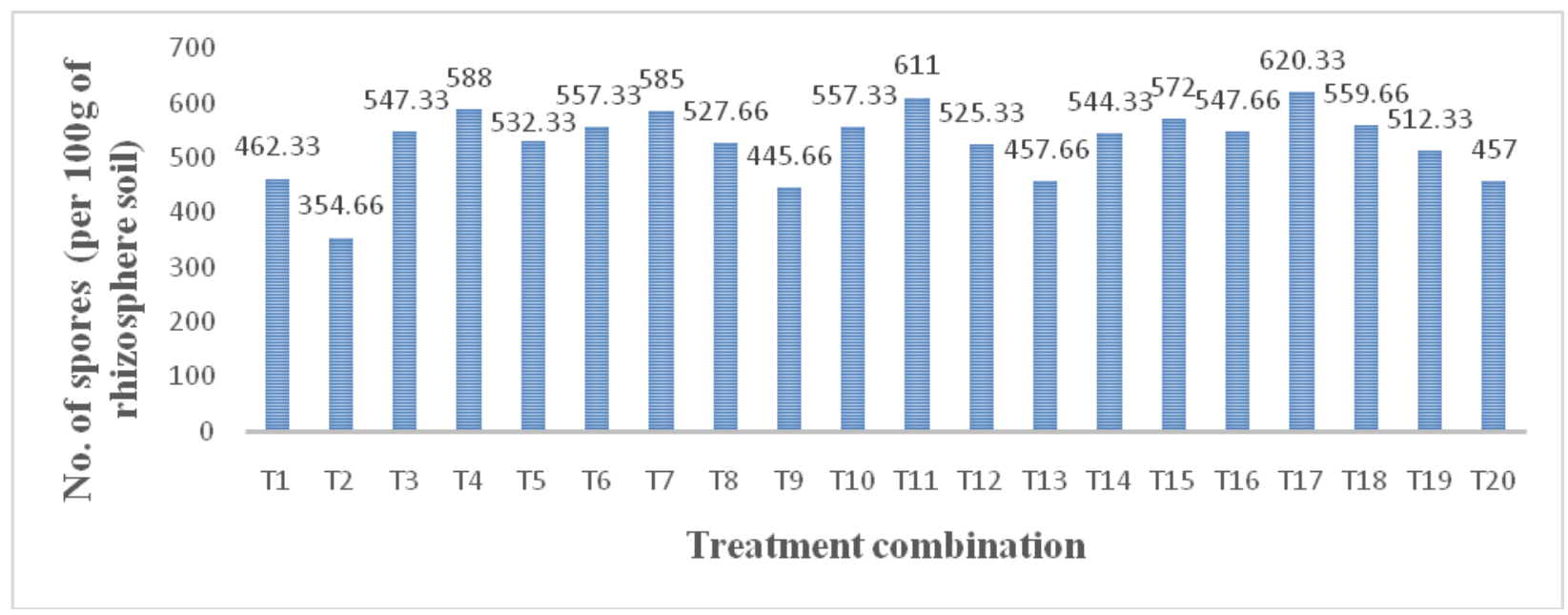

Fig.8 Number of spores per 100g of rhizosphere soil in Trap Crop Cycle-III

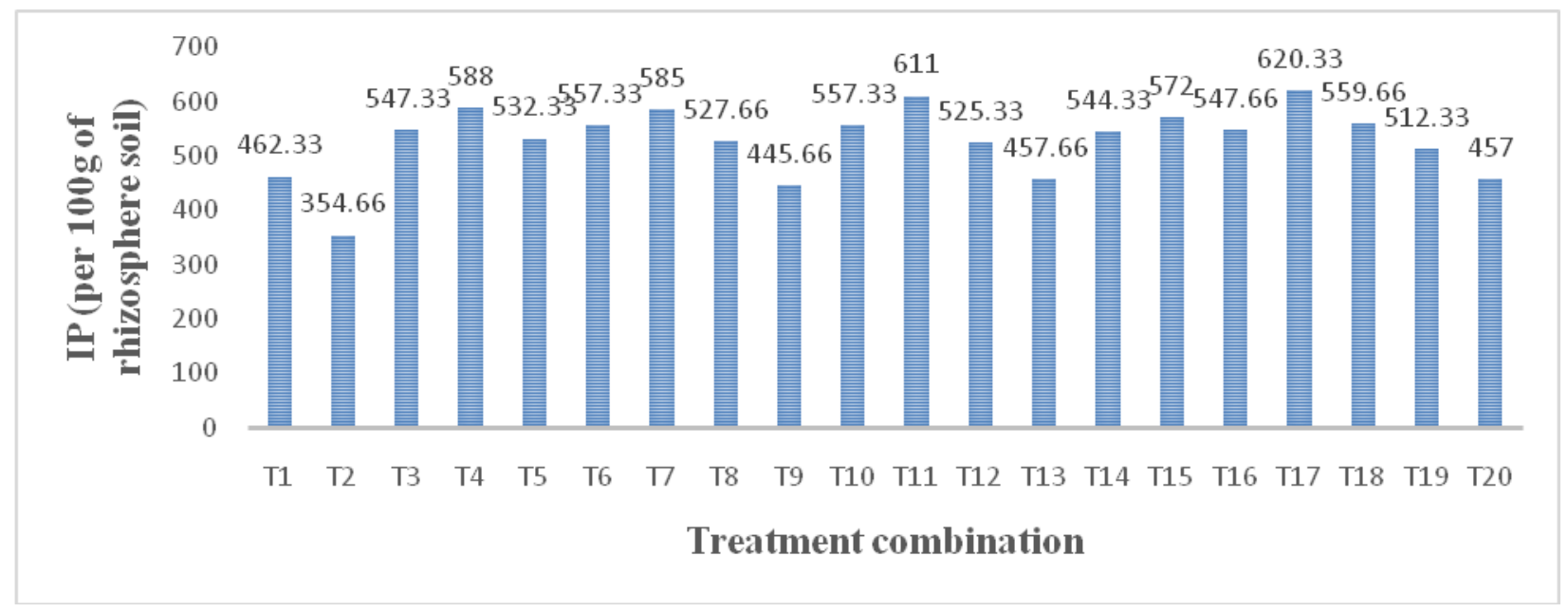

Fig.9 Infectious propagules of AMF per 100 gram of rhizosphere soil in Trap Crop Cycle-III 
Hawkins and George (1997) had used Sorghum bicolor as plant host for AM fungal propagule production in substrate-free cultivation by hydroponics method.

Gaur and Adholeya (2000) used Zea mays as plant host for the production of AM fungi which produce about $100 \mathrm{IP} / \mathrm{g} / \mathrm{substrate}$.

The infective propagules (IP) population of AMF ranged between 26.3-33.1 IP/g in natural soil in a study carried out by Maiti et al., (2009)

These variations may be attributed to soil moisture content in treatment plots, soil $\mathrm{P}$ levels, organic carbon and soil pH (Kaushal, 2000). However, there was continuous increase in IP numbers per 100 gram of rhizosphere (Boswell et al., 1998)

\section{Acknowledgement}

I express the depth of gratitude to, my Research Guide and Chairman of my Advisory Committee Dr.C.D. Deokar, Professor, College of Agriculture, Dhule. I am obligated to all the researchers and scientists whose literature helped me throughout the course of the investigation and to prepare this manuscript properly.

\section{References}

Boswell, E.P., Koide, R.T., Shumway, D.L. and Addy, H.D. 1998. Winter wheat cover cropping, VA mycorrhizal fungi and maize growth and yield. Agric. Ecosys. Environ., 67: 55-65.
Gaur, A. and Adholeya, A. 2000. Effect of the particle size of soil- less substrates upon AM fungus inoculum production. Mycorrhiza, 10(1): 43-48.

Hawkins, H.J. and George, E. 1997. Hydroponic culture of the mycorrhizal fungus Glomus mosseae with Linum usitatissimum L. Sorghum bicolor L. and Triticum aestivum L. Plant Soil, 196: 143-149.

Harley, J.L. 1959. The Biology of Mycorrhiza. Interscience Publishers, Inc. New York. Pp. 234.

Kadian Nisha, Yadav Kuldeep and Aggarwal Ashok. 2018. Mass multiplication of arbuscular mycorrhizal fungi associated with some leguminous plants: an ecofriendly approach. Indian J. Exp. Biol., 56: 258-266.

Kaushal Sangeeta, 2000. Influence of edaphic factor on VA Mycorrhizal fungal spore population and root colonization in Acacia nilotica in Rajasthan. J. Mycol. And Pl. Pathol. 30(3): 380-388.

Maiti, D., Barnwal, M.K., Singh, R.K. and Variar, M. 2009. A new protocol for onfarm production of arbuscular mycorrhizal mass inoculum for rained upland rice. Indian Phytopath., 62(1): 31-36.

Sreenivassa, M.N. 1992. Selection of an efficient vesicular-arbuscualr mycorrhizal fungus for Chilli (Capsicum annuum L.) Sci. Hortic., 50: 53-58.

Verma, V.K. 2011. Studies on on-farm production techniques of arbuscular mycorrhizal fungi. M.Sc. (Agri.) thesis submitted to MPKV, Rahuri.

\section{How to cite this article:}

Raut, S.B., C.D. Deokar, A.M. Navale and Dahatonde. 2019. On-farm Production of Arbuscular Mycorrhizal (AM) Fungi Using Trap Crop Cycles. Int.J.Curr.Microbiol.App.Sci. 8(10): 1084-1101. doi: https://doi.org/10.20546/ijcmas.2019.810.128 